\title{
Diagnóstico De La Situación Educativ - Laboral De Los Jóvenes De 18 A 25 Años De Las Parroquias Rurales Del Cantón Ambato - Ecuador
}

\author{
Diagnosis Of Educational Situation - Labour Of Youth With Ages \\ From 18 To 25 Years From Rural Parishes Of Ambato Canton, \\ Ecuador
}

María del Carmen Gómez Romo1, Andrés F. López Gómez².

\begin{abstract}
1 - Magister en Gerencia Empresarial, MBA, mención en Proyectos, por la Escuela Politécnica Nacional. Docente de la Facultad de Contabilidad y Auditoría de la Universidad Técnica de Ambato. mariadgomez@uta.edu.

2 - Magister en Administración de Empresas, mención Planeación por la Pontificia Universidad Católica del Ecuador sede Ambato. Docente en la Facultad de Ciencias Administrativas de la Universidad Técnica de Ambato. af.lopez@uta.edu.ec
\end{abstract}

\section{Resumen}

En esta investigación se describe la situación educativo - laboral de los jóvenes de 18 a 25 años de las parroquias rurales del cantón Ambato, a partir de una muestra de 313 jóvenes elegida aleatoriamente en forma proporcional en cada parroquia, para proveer de información útil a la de toma de decisiones en el ámbito educativo y político, con el fin de contribuir a la formación de mano de obra calificada que sirva de apoyo a los sectores productivos. De la investigación se desprende que la situación tanto educativa como laboral de los jóvenes es elemental, pues solamente el 11,8 \% tienen educación superior; además, la situación laboral de los jóvenes es precaria, el 71,8\%, de los jóvenes que trabajan manifiestan que sus ingresos no cubren completamente sus necesidades, lo que se agrava con la inestabilidad laboral, dado que el 32,1\% tienen trabajo eventual. Esta situación demanda de manera urgente el diseño e implementación de planes y programas de formación y capacitación profesional que contribuyan a potenciar a la población joven, económicamente activa a fin de que pueda insertarse de manera digna en el mercado laboral. A partir de este estudio se identifican buenas perspectivas para el planteamiento de propuestas educativas en el sector de confecciones y de servicios en virtud de la preferencia expresada por los jóvenes y de su presencia en los sectores productivos.

Palabras claves: Jóvenes, parroquias rurales, educación, trabajo, mano de obra.

\section{Abstract}

In this research the educational situation described - employment of young people aged 18 to 25 years of rural parishes of the canton Ambato, from a sample of 313 young people chosen randomly proportionally in each parish, to provide useful information to the decision-making in the educational and political spheres, in order to contribute to the training of skilled labor that will support the productive sectors. Research shows that the educational situation both as a labor of young people 
is elementary, as only $11.8 \%$ have higher education; In addition, the employment situation of young people is precarious, $71.8 \%$ of young workers report that their revenues do not fully cover their needs, which is compounded by job insecurity, since $32.1 \%$ have jobs eventual. This situation urgently demand the design and implementation of plans and programs of vocational education and training that help empower economically active so that it can be inserted in a dignified way in the labor market young people. From this study good prospects for the educational approach proposed in the apparel sector and services under the preference expressed by young people and their presence in the productive sectors are identified.

Keywords: Youth, rural parishes, education, employment, work force.

\section{Introducción.}

Ecuador sufrió una profunda crisis en la última década del siglo XX que se reflejó en los altos niveles de concentración de la riqueza en ciertos sectores sociales (Álvarez, 2007). Por otro lado el deterioro de las condiciones de vida de la mayoría de su población, dejando evidentes secuelas de analfabetismo, marginalidad, desempleo y subempleo, (Ordoñez, 2010), repercutió en la creciente insatisfacción de las necesidades básicas y la reducida disponibilidad de ingresos que aqueja a los sectores menos favorecidos.

En el centro del país se encuentra la provincia de Tungurahua (Zona 3) que describe una intensa actividad comercial y productiva. Su cabecera cantonal es Ambato, dividida en 10 parroquias urbanas y 18 parroquias rurales, con una población de 326.688 habitantes que representa el $65,4 \%$ de la población de la provincia, con una concentración del $50,1 \%$ en el área urbana y el 49,9\% en el área rural.
(Instituto Ecuatoriano de Estadísticas y Censos-INEC, 2010). El área rural es considerada como una región vulnerable dadas las limitadas condiciones de vida, en virtud de lo cual, la presente investigación se centra en la población entre los 18 y 25 años de estas parroquias del cantón Ambato.

\section{Objetivo.}

Describir la situación educativa y laboral de los jóvenes de 18 a 25 años de las parroquias rurales del cantón Ambato, a fin de proporcionar información para la elaboración de propuestas educativas, políticas públicas y el impulso de nuevas investigaciones.

\section{Antecedentes.}

En el año 2.000, en Ecuador se implantó el esquema de dolarización como una salida desesperada a la crisis que imperaba (Larrea, 2004). Con este esquema monetario y al fijar un tipo de cambio de 25.000 sucres por dólar (Registro Oficial, 2000), se produjo un ajuste de 
precios de bienes y servicios que desencadenó un proceso inflacionario sin precedentes en la historia nacional. La desconfianza en el sistema financiero provocó un cambio en las preferencias de consumo público, que dirigió todo su ahorro a la adquisición de bienes tangibles como vivienda, vehículo y electrodomésticos.

La provincia de Tungurahua ubicada en la región sierra en el centro del Ecuador, tiene una superficie aproximada de $3.400 \mathrm{~km}^{2}$, una población de 514.354 habitantes, una densidad poblacional de 149,75 habitantes por $\mathrm{km}^{2}$, en donde $43 \%$ de la población se encuentra en la zona rural, mientras que el $57 \%$ está en las zonas urbanas (INEC, 2010). Su tasa de crecimiento de estudiantes matriculados en el nivel básico y bachillerato es del 3,4\%, en el periodo 2008 a 2014, (OBEST, 2015). Según la Ley Orgánica de Educación Intercultural, la Educación General Básica está compuesta por 10 años de cursado obligatorio, a partir de los 5 años de edad, que continúa con el Bachillerato que comprende 3 años de educación (Registro Oficial, 2011).
En este escenario una de las variables macroeconómicas más afectadas por la crisis es el empleo, considerando que, desde 1962 la población económicamente activa (PEA), ha cambiado sustancialmente pasando de agrícola a manufacturera (Larrea, C. Larrea, A. Maldonado, 2009). Las tasas de ocupación, subempleo y desempleo en las ciudades han evolucionado por lo que es importante reflexionar acerca de este fenómeno social a fin de buscar perspectivas educacionales para el desarrollo de acuerdo a lo que expresa en la Tabla No 1 .

Tabla $\mathbf{N}^{\circ}$ 1. Tasa de ocupación plena en las principales ciudades del Ecuador.

\begin{tabular}{|l|lllllll|}
\hline \multicolumn{1}{|c}{} & mar-08 & mar-09 & mar-10 & mar-11 & mar-12 & mar-13 & mar-14 \\
\hline \hline Quito & $54,00 \%$ & $46,90 \%$ & $49,50 \%$ & $55,70 \%$ & $60,10 \%$ & $60,10 \%$ & $67,60 \%$ \\
Guaya quil & $41,60 \%$ & $35,40 \%$ & $38,80 \%$ & $42,10 \%$ & $53,00 \%$ & $52,80 \%$ & $54,60 \%$ \\
Cuenca & $50,50 \%$ & $53,10 \%$ & $50,40 \%$ & $52,30 \%$ & $60,60 \%$ & $64,70 \%$ & $60,10 \%$ \\
Ambato & $36,60 \%$ & $36,90 \%$ & $45,20 \%$ & $52,80 \%$ & $53,90 \%$ & $41,50 \%$ & $45,40 \%$ \\
\hline
\end{tabular}

Fuente: Instituto Nacional de Estadísticas y Censos INEC. Indicadores Laborales Marzo 2014. 
Tabla $\mathbf{N}^{\circ}$ 2. Tasa de subempleo en las principales ciudades del Ecuador.

\begin{tabular}{|l|r|r|r|r|r|r|r|}
\hline & mar-08 & mar-09 & mar-10 & mar-11 & mar-12 & mar-13 & mar-14 \\
\hline \hline Quito & $35,30 \%$ & $43,80 \%$ & $40,20 \%$ & $35,20 \%$ & $32,80 \%$ & $31,70 \%$ & $26,90 \%$ \\
\hline Guayaquil & $48,70 \%$ & $49,90 \%$ & $46,20 \%$ & $46,10 \%$ & $39,60 \%$ & $39,80 \%$ & $38,70 \%$ \\
\hline Cuenca & $43,50 \%$ & $41,10 \%$ & $44,70 \%$ & $43,10 \%$ & $33,80 \%$ & $31,30 \%$ & $36,40 \%$ \\
\hline Ambato & $57,30 \%$ & $58,00 \%$ & $50,50 \%$ & $42,80 \%$ & $41,00 \%$ & $50,80 \%$ & $48,80 \%$ \\
\hline
\end{tabular}

Fuente: Instituto Nacional de Estadísticas y Censos INEC. Indicadores Laborales Marzo 2014.

Tabla $\mathbf{N}^{\circ}$ 3. Tasa de desempleo en las principales ciudades del Ecuador.

\begin{tabular}{|l|rrrrrrr|}
\hline & mar-08 & mar-09 & mar-10 & mar-11 & mar-12 & mar-13 & mar-14 \\
\hline \hline Quito & $6,50 \%$ & $7,20 \%$ & $7,20 \%$ & $5,70 \%$ & $3,70 \%$ & $4,10 \%$ & $4,30 \%$ \\
Guayaquil & $8,00 \%$ & $14,10 \%$ & $12,30 \%$ & $10,00 \%$ & $6,30 \%$ & $5,50 \%$ & $6,10 \%$ \\
Cuenca & $5,00 \%$ & $4,90 \%$ & $3,80 \%$ & $4,10 \%$ & $4,70 \%$ & $3,20 \%$ & $3,20 \%$ \\
Ambato & $4,50 \%$ & $4,10 \%$ & $3,10 \%$ & $3,50 \%$ & $4,40 \%$ & $4,40 \%$ & $5,80 \%$ \\
\hline
\end{tabular}

Fuente: Instituto Nacional de Estadísticas y Censos INEC. Indicadores Laborales Marzo 2014.

Es notorio que existe una diferencia de 1,4 puntos porcentuales de marzo del 2013 a marzo del 2014, lo que indica el deterioro de la situación laboral en la ciudad de Ambato.

Al mes de Junio del 2015, la tasa de empleo adecuado se situó en 45,90\%; mientras que la tasa de empleo inadecuado, se registró un $49,07 \%$ y la de desempleo en 4,47\%; cabe señalar que se entiende por empleo inadecuado a personas con empleo que no satisfacen las condiciones mínimas de horas o ingresos laborales (subempleados por insuficiencia de tiempo de trabajo o subempleados por insuficiencia de ingresos). Además se determinó que entre Junio 2014 y Junio 2015, la tasa de empleo adecuado disminuye en 2,91\%, mientras que la tasa de empleo inadecuado se incrementa en $2,79 \%$, con diferencias significativas al $95 \%$ de confianza, (INEC, 2015). Lo anteriormente expresado deja en claro que casi la mitad de la población en el Ecuador no tiene condiciones adecuadas de empleo y que esta situación ha desmejorado alrededor de un $3 \%$ en el año 2015 tanto en empleo adecuado como en empleo inadecuado se refiere. Por otro lado al analizar las cifras que reporta el INEC con respecto al desempleo y subempleo en Ambato, se observa que en el 2015, mientras decrece el desempleo, con respecto al 2014, se incrementa el subempleo, lo que evidentemente ocurre por cuanto las personas desempleadas pasan a ser subempleadas sin que ello signifique un mejoramiento de su situación laboral (Ordoñez, 2010). Así pues, la simple comparación cuantitativa no es útil sino que es necesario el análisis del fenómeno social con la asociación de las variables involucradas. 
El problema recurrente de subempleo y desempleo que sufre el Ecuador tiene raíces de diversa índole que no se las puede enmarcar solo en una de las áreas de la vida nacional, ni atribuir a determinados actores por el mero hecho de ocupar cierta posición socioeconómica o desarrollar una determinada actividad. Así, en la Agenda para el Desarrollo, publicada por el exConsejo Nacional de Desarrollo se incorporan de forma resumida las siguientes causas:

Inflexibilidad y difícil concepción de las leyes laborales.

$\checkmark$ Inapropiada orientación económica y social, que impidió el desarrollo de la inversión y la generación de empleo.

Falta de correspondencia entre el sistema educativo y las necesidades de la economía. La educación y formación de los recursos humanos no han respondido de forma adecuada a las demandas y necesidades del aparato productivo y del sistema económico global.

$\checkmark$ Insuficiencia de políticas específicas de empleo y deficiente apoyo a programas que respondan a los requerimientos de los sectores productivos.

$\checkmark$ Elevado ritmo de crecimiento demográfico y reducidos periodos de vida escolar caracterizados por una alta deserción y baja continuidad entre niveles educativos, que provocan una sobreoferta de mano de obra, especialmente joven, en el mercado laboral.

$\checkmark$ Crisis de la economía campesina e inadecuadas condiciones de producción agrícola que contribuyen a agudizar la migración campo-ciudad, aumentando la presión por la búsqueda de empleo en el área urbana (CONADE, 1994, pág. 2).

En tales condiciones, las estrategias más adecuadas para salir de la crisis son aquellas que operan sobre los esfuerzos de innovación en materia de gestión del talento humano y gestión del conocimiento (Calderón, Álvarez \&Naranjo, 2006). La importancia de esta gestión radica en el hecho de que al potenciar en forma efectiva los factores interactuantes, el resultado determina la eficacia, posicionamiento y nivel de competitividad en las organizaciones.

El Gobierno Nacional de la República del Ecuador pone de manifiesto la importancia de la "Revolución productiva a través del conocimiento y el talento humano"; en la primera edición del Folleto Informativo 2012, se expresa el ambicioso reto de transformar la matriz productiva, que propone un modelo democrático e incluyente, basado en el conocimiento y las capacidades de las y los ecuatorianos (SENPLADES, 2012). En este sentido, se establece que es el conocimiento el elemento discriminante entre hacer las cosas de la misma manera y hacerlas de un modo 
diferente con lo cual a no dudarlo se aspiraría a obtener resultados distintos (Fleitas, 2002).

La Agenda Tungurahua 2015-2017 (2011), describe a la provincia como principalmente agrícola $(26,94 \%$ de la población económicamente activa), manufacturera $(18,63 \%$ de la PEA) y comercial al por mayor y menor $(16,55 \%$ de la PEA), servicios varios $(14,22 \%$ de la PEA) y actividades como transporte, construcción, servicios financieros y otros, distribuidas en proporciones menores. Dentro de sus objetivos provinciales se encuentra el incremento del ingreso para cuyo efecto se han definido políticas de fomento productivo que a través de La Estrategia Agropecuaria, la Agenda de Turismo, Agenda de Competitividad y la Agenda Artesanal han direccionado las inversiones del sector público y privado.

Tungurahua, en el año 2014, decidió intensificar la matriz productiva, en virtud de sus fortalezas en los ámbitos productivo, agropecuario, comercial e industrial, teniendo como fortaleza la demostrada capacidad de sus habitantes para reponerse antes las crisis causadas por catástrofes naturales ocurridas en el pasado, como los terremotos y erupciones volcánicas. La generación de ingresos y generación de fuentes de trabajo tiene como base la transformación y generación de nuevos productos, la sustitución de importación y maximización de exportaciones (Agenda
Tungurahua, 2011). Reviste gran importancia entonces, transitar de ser productores de materias primas a productores de productos terminados creando valor agregado y optimizando recursos, de esta manera se propende a la mejora la producción y la productividad de las empresas de Tungurahua.

Se considera importante realizar el diagnóstico de la situación educativa-laboral de los jóvenes de 18 a 25 años de las parroquias rurales del cantón Ambato por considerar que este segmento vulnerable de la población constituye la potencial mano de obra calificada para los sectores productivos y porque la educación debe ser capaz de contribuir a la formación del individuo de tal manera que a través del proceso educativo logre estrechas relaciones con el entorno social y productivo de su localidad e integre definitivamente el estudio con el trabajo. Existen retos que van más allá de la mera adaptación de las estructuras y exigencias de capacidades directivas, es necesario predisposición al cambio, lo que podría lograrse a través de una formación y capacitación efectiva del talento humano, adaptada a necesidades reales (Mertens, 1997).

\section{Metodología.}

Esta es una investigación cualicuantitava de tipo descriptivo. Las unidades de observación fueron los jóvenes de 18 a 25 años habitan en las parroquias rurales 
del cantón Ambato-Ecuador. El universo estuvo constituido por 21.572 jóvenes, según el VII Censo de Polación y VI de Vivienda. Ecuador (REDATAM, 2012), de donde se extrajo una muestra aleatoria de 313 jóvenes, calculado para población finita con el 95\% de confianza y el 5,5\% de error (Webster, 1999), que estuvo distribuida en grupos etarios, de la siguiente manera: 18 años (11,5\%), 19 años (13,1\%), 20 años (9,3), 21 años (8,6\%), 22 años (9,9\%), 23 años (18,5\%), 24 años (10,5\%), 25 años (18,5\%), de los cuales participaron 132 mujeres $(42,2 \%)$ y 181 hombres $(57,8 \%)$, a la que se aplicó un cuestionario estructurado de 19 items, algunos con escala nominal y otros con escala ordinal de tres niveles de acuerdo al caso, mismo que fue validado previamente a través de pruebas piloto. El procesamiento y análisis de datos se hizo con el paquete estadístico SPSS - Statistical Package for the Social Sciences (Arbuckle, J. L. 2010).

\section{Resultados.}

La primera descripción se hace con relación al estado laboral; así, de los 313 jóvenes, el $78.91 \%$ se encontraban trabajando al momento en que fueron encuestados; mientras que el $21,09 \%$ no estaban trabajando. De este último grupo, 33 jóvenes nunca antes han trabajado, quienes consideraron que la razón es porque no hay trabajo $(49,2 \%)$ o porque no están preparados para ejercer un puesto de trabajo (50,8\%). No obstante, el 41,1\% manifestó haber estado preparado para desempeñar un trabajo, en contraste con el 58,9\% que está consciente de que no está preparado.

$\mathrm{Al}$ analizar los 280 casos de los jóvenes que se encontraban trabajando o habían trabajado alguna vez, se estableció que el 66,8\% había superado el primer año de trabajo, por lo que ellos consideraron tener una ocupación permanente; el $32,10 \%$ eventual, y el 1,1\% no supieron identificar su estabilidad laboral. Por otro lado, con respecto a la manera en que aprendieron su oficio se encontró que, un grupo aprendió como "Aprendiz" en alguna empresa $(56,1 \%)$, otros aprendieron dentro de su núcleo familiar (22,5\%), y finalmente el último grupo aprendió en algún centro de estudios (21,4\%), como se presenta en el siguiente tabla. 
Tabla $N^{\circ}$ 4. Situación laboral de los jóvenes de las parroquias rurales de Ambato.

\begin{tabular}{|c|c|c|c|c|c|c|}
\hline & \multirow{2}{*}{\multicolumn{2}{|c|}{ ¿Actualmente se encuentra trabajando? }} & \multicolumn{3}{|c|}{ Estabilidad laboral } & \multirow{2}{*}{ Total } \\
\hline & & & Permanente & Eventual & Otro & \\
\hline \multirow{4}{*}{ Si } & \multirow{3}{*}{ Modo en que aprendió el oficio } & Aprendiz & $44,10 \%$ & $12,60 \%$ & $0 \%$ & $56,70 \%$ \\
\hline & & Tradición familiar & $13,40 \%$ & $8,50 \%$ & $0 \%$ & $21,90 \%$ \\
\hline & & Estudió en un centro & $13,80 \%$ & $6,90 \%$ & $0,80 \%$ & $21,50 \%$ \\
\hline & Total & & $71,30 \%$ & $27,90 \%$ & $0,80 \%$ & $100,00 \%$ \\
\hline \multirow{4}{*}{ No } & \multirow{3}{*}{ Modo en que aprendió el oficio } & Aprendiz & $12,10 \%$ & $36,40 \%$ & $3 \%$ & $51,50 \%$ \\
\hline & & Tradición familiar & $6,10 \%$ & $21,20 \%$ & $0 \%$ & $27,30 \%$ \\
\hline & & Estudió en un centro & $15,20 \%$ & $6,10 \%$ & $0,00 \%$ & $21,20 \%$ \\
\hline & Total & & $33,30 \%$ & $63,60 \%$ & $3,00 \%$ & $100,00 \%$ \\
\hline \multirow{4}{*}{ Total } & \multirow{3}{*}{ Modo en que aprendió el oficio } & Aprendiz & $40,40 \%$ & $15,40 \%$ & $0,4 \%$ & $56,10 \%$ \\
\hline & & Tradición familiar & $12,50 \%$ & $10,00 \%$ & $0 \%$ & $22,50 \%$ \\
\hline & & Estudió en un centro & $13,90 \%$ & $6,80 \%$ & $0,70 \%$ & $21,40 \%$ \\
\hline & \multicolumn{2}{|l|}{ Total } & $66,80 \%$ & $32,10 \%$ & $1,1 \%$ & $100,00 \%$ \\
\hline
\end{tabular}

Fuente: Investigación de campo (2015)

La satisfacción de necesidades con los ingresos percibidos tiene la ponderación más alta en la categoría intermedia en casi todos los niveles de educación. El nivel de educación oscila entre los niveles de Educación Básica $\left(1^{\circ}\right.$ a $10^{\circ}$ año), bachillerato $y$ superior $\mathrm{o}$ universitario, del que el grupo más grande es aquel que ha cursado al menos algún nivel de bachillerado en contraste con el grupo que ha cursado algún nivel de educación superior que es el más pequeño.

Tabla $\mathrm{N}^{\circ}$ 5. Satisfacción de necesidades con los ingresos percibidos por los jóvenes.

\begin{tabular}{|c|c|c|c|c|}
\hline \multirow{2}{*}{ Nivel de estudios } & \multicolumn{4}{|c|}{ Nivel de satisfacción de necesidades con los ingresos } \\
\cline { 2 - 5 } & Completamente & Medianamente & Mínimamente & Total \\
\hline \hline $\mathbf{1}^{\circ} \mathbf{a} \mathbf{3}^{\circ}$ & $1,1 \%$ & $0,7 \%$ & $0,4 \%$ & $2,1 \%$ \\
\hline $\mathbf{4}^{\circ} \mathbf{a} \mathbf{7}^{\circ}$ & $6,1 \%$ & $10,0 \%$ & $3,2 \%$ & $19,3 \%$ \\
\hline $\mathbf{8}^{\circ} \mathbf{a} \mathbf{1 0}^{\circ}$ & $1,4 \%$ & $7,1 \%$ & $2,1 \%$ & $10,7 \%$ \\
\hline $\mathbf{1}^{\circ} \mathbf{a ~ 3}^{\circ} \mathbf{B a c h i l l e r a t o}^{\mathbf{U n}}$ & $16,4 \%$ & $32,9 \%$ & $6,8 \%$ & $56,1 \%$ \\
\hline Total & $3,2 \%$ & $7,1 \%$ & $1,4 \%$ & $11,8 \%$ \\
\hline
\end{tabular}

Fuente: Investigación de campo (2015)

Resulta interesante identificar la autopercepción de los jóvenes, dado que, es necesario un contar con un nivel de conciencia para buscar cambios de perspectivas en una realidad. Se encontró que los jóvenes perciben de modo más alto sus habilidades y destrezas que sus conocimientos, lo que oscilan entre un nivel alto y medio, mientras que, son muy pocos los que los han elegido el nivel bajo. 
Tabla $\mathrm{N}^{\circ}$ 6. Autopercepción de conocimientos y habilidades en el desempeño laboral

\begin{tabular}{|c|c|c|c|c|c|c|c|c|}
\hline \multirow{2}{*}{ Nivel de estudios } & \multicolumn{4}{|c|}{ Conocimientos } & \multicolumn{4}{|c|}{ Habilidades y destrezas } \\
\hline & Alto & Medio & Bajo & Total & Alto & Medio & Bajo & Total \\
\hline \multirow{2}{*}{$1^{\circ}$ a $3^{\circ}$} & 4 & 2 & 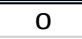 & 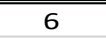 & 3 & 3 & 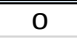 & 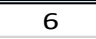 \\
\hline & $1,4 \%$ & $0,7 \%$ & $0,0 \%$ & $2,1 \%$ & $1,1 \%$ & $1,1 \%$ & $0,0 \%$ & $2,1 \%$ \\
\hline \multirow{2}{*}{$4^{\circ}$ a $7^{\circ}$} & 28 & 22 & 2 & 54 & 28 & 24 & 2 & 54 \\
\hline & $10,0 \%$ & $7,9 \%$ & $1,4 \%$ & $19,3 \%$ & $10,0 \%$ & $8,6 \%$ & $0,7 \%$ & $19,3 \%$ \\
\hline \multirow{2}{*}{$8^{\circ}$ a $10^{\circ}$} & 12 & 17 & 1 & 30 & 13 & 16 & 1 & 30 \\
\hline & $4,3 \%$ & $6,1 \%$ & $0,4 \%$ & $10,7 \%$ & $4,6 \%$ & $5,7 \%$ & $0,4 \%$ & $10,7 \%$ \\
\hline \multirow{2}{*}{$1^{\circ}$ a $3^{\circ}$ Bachillerato } & 70 & 86 & 1 & 157 & 90 & 66 & 1 & 157 \\
\hline & $25,0 \%$ & $30,7 \%$ & $0,4 \%$ & $56,1 \%$ & $32,1 \%$ & $23,6 \%$ & $0,4 \%$ & $56,1 \%$ \\
\hline \multirow{2}{*}{ Universitario } & 17 & 16 & 0 & 33 & 20 & 13 & 0 & 33 \\
\hline & $6,1 \%$ & $5,7 \%$ & $0,0 \%$ & $11,8 \%$ & $7,1 \%$ & $4,6 \%$ & $0,0 \%$ & $11,8 \%$ \\
\hline \multirow{2}{*}{ Total } & 131 & 143 & 6 & 280 & 154 & 122 & 4 & 280 \\
\hline & $46,8 \%$ & $51,1 \%$ & $2,1 \%$ & $100,0 \%$ & $55,0 \%$ & $43,6 \%$ & $1,4 \%$ & $100,0 \%$ \\
\hline
\end{tabular}

Fuente: Investigación de campo (2015)

Al indagar con relación a las perspectivas que los jóvenes tienen a cerca de estudiar para prepararse para desempeñar eficientemente un puesto de trabajo, se determinó que el 90,4\% desea hacerlo, frente al 5,1\% que no desea estudiar y el 4,5\% que está indeciso.
Comparando la proporción de jóvenes que trabajaban en determinado sector productivo al momento de la investigación, con su preferencia de trabajo futuro, se encontró que el sector de servicios y de confecciones son los de mayor interés, así es que se debería centrar la atención en aquellos, para elaborar una planificación educativa con miras a los potenciales educandos.

Gráfico 1. Trabajo actual vs. Trabajo futuro en Sectores Productivos.

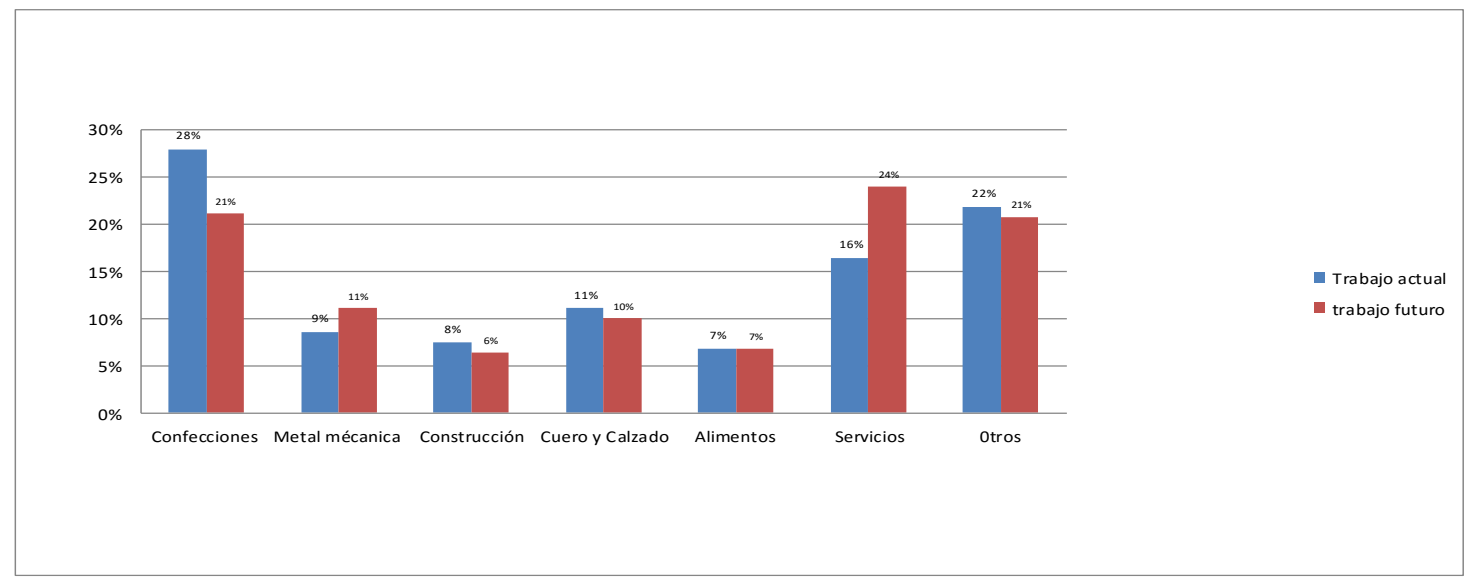

Fuente: Investigación de campo (2015)

Además, esta investigación proporciona información relativa al tipo de entidad donde desearían estudiar los jóvenes, así se tiene que el $55 \%$ prefiere estudiar en un instituto superior, 
el $30,4 \%$ en un colegio técnico y solamente el $14,7 \%$ preferiría estudiar en la misma empresa donde trabaja, abriéndose oportunidades para la construcción de programas educativos en especial en institutos superiores, teniendo muy pocas probabilidades de éxitos los programas educativos al interior de empresas. Definitivamente, es imprescindible contar con programas de educación que permitan formar en los jóvenes las competencias necesarias, para que se conviertan en personas idóneas para los distintos puestos de trabajo, pues el Plan del Buen Vivir propende a que los receptores de ingresos en los hogares ambateños y ecuatorianos estén ubicados en una posición contractual, (SENPLADES, 2013).

\section{Conclusiones}

El Ecuador necesita tener una producción diversificada de bienes con valor agregado, para ello, se hace imperativo que se forme al talento humano de tal manera que adquieran las competencias necesarias a partir de conocimientos que permitan desarrollar nuevas y eficientes maneras de hacer las cosas, más aún si existe la conciencia de que la mano de obra calificada constituye una piedra angular donde se deben apoyar los sectores productivos, como lo corrobora Sánchez (2005).
Entre las acciones que se presentan como factibles se menciona la necesidad de implementar un sistema de formación del talento humano, en función del mercado laboral; profundizar la vinculación entre la política y la planificación para el desarrollo; fortalecer la participación popular en la determinación de las necesidades y propuesta de alternativas de solución para el desempleo como la creación de programas tanto de formación como de capacitación bien direccionados.

El 46,8 \% de jóvenes manifiestan estar satisfechos, el 47,5\% poco satisfechos con su trabajo, y el 5,71\%, expresa su disconformidad, fenómeno que se explica por el conformismo propio de este segmento, caracterizado por la falta de oportunidades y deseos de superación, lo que se contrasta por otro lado, con la limitada satisfacción de necesidades con los ingresos fruto de su trabajo, por esta razón resulta imprescindible brindar a los jóvenes un nuevo enfoque que abrigue perspectivas más alentadoras para hacer frente a los retos que, a no dudarlo, la vida les pondrá a su paso.

Finalmente, es relevante expresar la importancia de llevar a cabo continuos planes de monitoreo y evaluación con el porpósito de determinar el alacance de metas y objetivos planteados en el tema educativo-laboral, haciendo continuos replanteamientos moficiaciones y desarrollando estrategias, tanto 
con la participación del sector público como del privado; pues, la educción siendo la base del desarrollo de los puebos, y la gente proactiva y trabajadora, el motor de las organizaciones, es posible crear sinergia y e incrementar el nivel de compectitividad de los sectores productivos.

\section{Referenias Bibliograficas}

1. ARBUCKLE, J. L. (2010). IBM SPSS Amos 19 user's guide. Crawfordville, FL: Amos Development Corporation, 635.

2. CALderón, G., Álvarez, C. M, \& Naranjo Valencia, J. C. (2006). Gestión bumana en las organizaciones un fenómeno complejo: Evolución, retos, tendencias $y$ perspectivas de investigación. Cuadernos de Administración, 19(32).

3. CONADE. Consejo Nacional de Dearrollo. (1994). Agenda de Desarrollo Quito, Ecuador: s/e. Quito-Ecuador.

4. GOBIERNO PROVINCIAL DE TUNGURAHUA (2015). Agenda Tungurabua 2015-2017, AmbatoEcuador, (19).

5. INEC. Instituto Nacional de Estadísticas y Censos. VII Censo de Polación

y VI de Vivienda. Ecuador. (2010).

6. INEC. Instituto Nacional de Estadísticas y Censos. (2015). Encuesta
Nacional de Empleo, Desempleo $y$ Subempempleo. Quito, Ecuador.

7. INEC. (2014). Instituto Nacional de Estadísticas y Censos. Indicadores Laborales. Quito, Ecuador. www. Ecuador encifras.gob.ec

8. LARREA, C., Larrea, A. I., \& Maldonado, P. (2009). Evolución de las condiciones sociales en Tungurabua: 19502006. Documento de trabajo UASB. QuitoEcuador.

9. LARREA, M. (2010). Dolarización y desarrollo bumano en Ecuador (Dossier). Quito-Ecuador.

10. LEGUIZAMÓN, S. A., \& Leguizamón, A. (2007). Concentración de la riqueza, millionarios y reproducción de la pobreza en América Latina. Sociologias,9(18).

11. MERTENS, L. (1997). Competencia laboral: sistemas, surgimiento y modelos. Montevideo: Organización Internacional del Trabajo (OIT), Centro Interamericano para el Desarrollo del Conocimiento en la Formación Profesional.

12. OBEST. (2015) Los niveles de educación, una perspectiva en la provincia de Tungurabua. Boletín coyuntura. No.7 (10). Ambato-Ecuador.

13. ORDOÑEZ, J. (2010). Empleo y migración en el Ecuador. Revista Fuente vol, 1(3).

14. ÓRGANO DEL GOBIERNO DEL ECUADOR. (2000). Registro Oficial No 63, 24 de Abril del 2000. 
15. ÓRGANO DEL GOBIERNO DEL ECUADOR. (2011). Registro Oficial $\mathrm{N}^{\circ}$ 417, 31 de marzo del 2011

16. REDATAM, Recuperación Datos para Áreas pequeñas por Microcomputador (2012). Recuperado de: http:/ / redatam.inec.gob.ec/cgibin/RpWebEn gine.exe $/$ EasyCross? \&BASE=DIEE2014 $\ll I T E M=C R U Z S I M P L E \& M A I N=W e$ bServerMain.inl

17. SÁNCHEZ DÍAZ, M. (2005). Breve inventario de los modelos para la gestión del conocimiento en las organizaciones. Acimed, 13(6), 0-0.

18. SANCHEZ-PARAMO, C. (2005). Pobreza en Ecuador. Breve, Banco Mundial.

19. SENPLADES, S. N. (2013). Plan Nacional de Desarrollo/Plan Nacional para el Buen Vivir 2013-2017. National Development Plan for Living Well, 2017.

20. WEBSTER, A. (1999). Estadística Aplicada a la Empresa y a la Economía. Bogotá, Colombia: McGraw Hill . 